Journal title: $\quad$ FAS

Article Number: $\quad 521832$

Dear Author/Editor,

Greetings, and thank you for publishing with SAGE. Your article has been copyedited, and we have a few queries for you. Please respond to these queries when you submit your changes to the Production Editor.

Thank you for your time and effort.

Please assist us by clarifying the following queries:

\begin{tabular}{|l|l|}
\hline No & \multicolumn{1}{|c|}{ Query } \\
\hline 1 & $\begin{array}{l}\text { Please check that (a) all authors are listed in the proper order; (b) clarify which part of each author's name is his or her } \\
\text { surname; (c) verify that all author names are correctly spelled/punctuated and are presented in a manner consistent with any } \\
\text { prior publications; and (d) check that all author information, such as affiliations and contact information, appears accurately. } \\
\text { [Barry J. O'Neill, Laura A. Sweeney, Paul J. Moroney, and Kevin J. Mulhall] }\end{array}$ \\
\hline 2 & $\begin{array}{l}\text { Please review the entire document for typographical errors, mathematical errors, and any other necessary corrections; check } \\
\text { headings, tables, and figures. }\end{array}$ \\
\hline 3 & $\begin{array}{l}\text { Please confirm that you have sufficiently reviewed your proof and queries, and that you understand this is your FINAL } \\
\text { opportunity to review your article before publication. }\end{array}$ \\
\hline 4 & \begin{tabular}{l} 
Please verify whether Figures 1 and 2 have been cited in the appropriate places. \\
\hline
\end{tabular} \\
\hline
\end{tabular}




\section{Atypical Stress-Avulsion Fracture of the Lisfranc Joint Complex}

Barry J. O'Neill, Laura A. Sweeney, Paul J. Moroney, and Kevin J. Mulhall[AQ: 1]

[AQ: 2][AQ: 3]
Abstract: Antiphospholipid syndrome and systemic erythematosus have been associated with metatarsal stress fractures. Stress fractures of the Lisfranc joint complex are uncommon injuries but have been reported to occur most frequently in ballet dancers. We present a case of an avulsion fracture of the Lisfranc joint complex that occurred spontaneously. We have reviewed the association between systemic conditions and metatarsal fractures and proposed a series of hypothetical pathological events that may have contributed to this unusual injury.

Levels of Evidence: Therapeutic, Level IV: Case report

Keywords: trauma; general disorders; fractures; sprains; strains; sports podiatry

\section{Introduction}

The tarsometatarsal (TMT) joint complex, commonly referred to as the Lisfranc joint, is named after a French surgeon who served in Napoleon's army. Jacques Lisfranc described an amputation involving the TMT joint secondary to a vascular injury that occurred when a rider fell from his horse with his foot caught in the stirrup. ${ }^{1,2}$ Injuries to the TMT joint complex are reported to account for approximately $0.2 \%$ of all fractures, ${ }^{3}$ but it has been suggested that up to $20 \%$ are missed on initial assessment. ${ }^{4}$ Low-energy trauma accounts for approximately one-third of all Lisfranc injuries, ${ }^{5}$ with athletic activity being the most common etiology. ${ }^{6}$ Stress fracture of the Lisfranc joint is uncommon but has been described predominantly in ballet dancers. ${ }^{7-10}$ We present a case of an avulsion type stress fracture involving the TMT joint complex in a 46-year-old female patient. This fracture differs from previous reports in that the patient was not involved in any form of sporting activity.

\section{Case Report}

A 46-year-old lady developed spontaneous pain in her left foot while walking. She described feeling a "pop" in her foot with immediate severe pain in the sole of her foot on weight bearing. She denied any history of injury or trauma to the foot. She did not seek medical attention initially but selfmedicated with simple over-the-counter analgesia.

Then, 6 weeks after the onset of symptoms, she attended accident and emergency (A\&E), and an anteroposterior and lateral $\mathrm{X}$ ray of her foot was performed (Figure 1). This X

DOI: 10.1177/1938640014521832. From the Department of Trauma and Orthopaedics, Mater Misericordiae University Hospital, Ireland. Address correspondence to Barry J. O'Neill, Department of Trauma and Orthopaedics, Mater Misericordiae University Hospital, Eccles Street, Dublin 7, Ireland; e-mail: barryoneill1922@gmail.com.

For reprints and permissions queries, please visit SAGE's Web site at http://www.sagepub.com/journalsPermissions.nav.

Copyright @ 2014 The Author(s) 


\section{Figure 1.}

A. Non-Weight-Bearing anteroposterior (AP) Radiograph Demonstrating Avulsion Type Fracture From Base of Second Metatarsal. B. Weight-Bearing AP Radiograph. C. Weight-Bearing Lateral Radiograph.
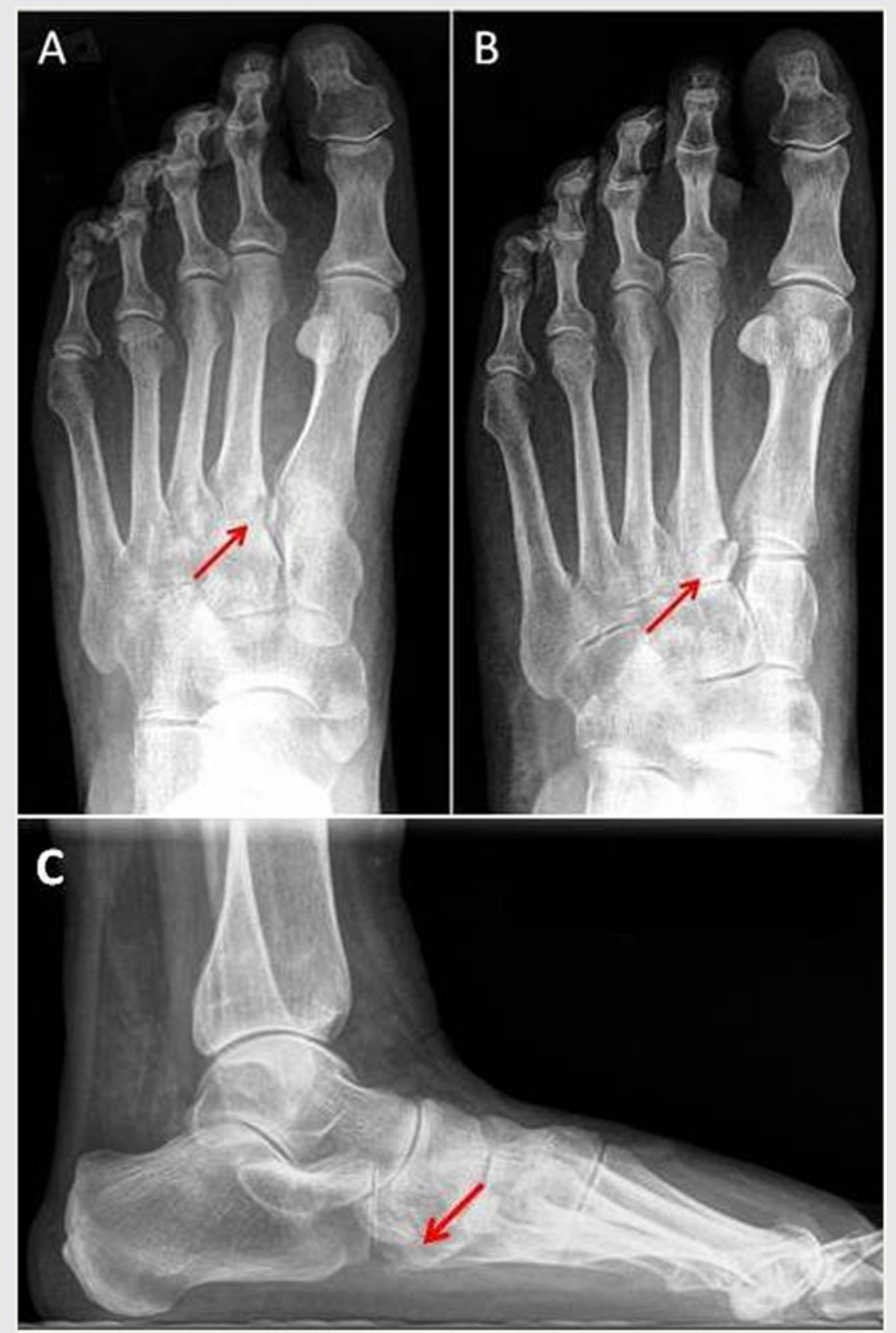

scan was performed. The CT scan clearly demonstrated an undisplaced fracture of the medial aspect of the base of the left second metatarsal. Clinically, the patient reported ongoing pain in her foot but was nontender to palpation and experienced no pain on bimanual

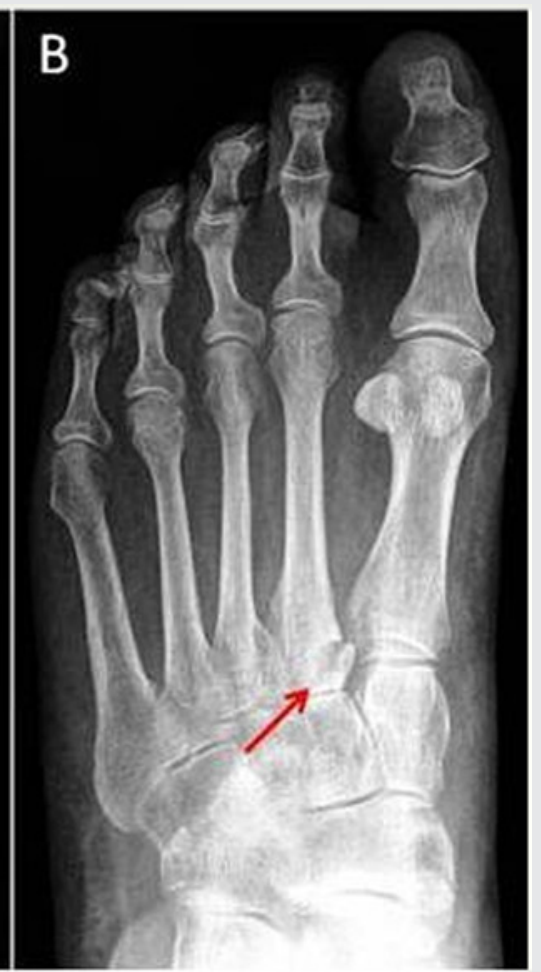

\section{Discussion}

The TMT joint complex is made up of bony and ligamentous structures designed to add support to the transverse arch of the foot. The anatomical arrangement of these structures makes the TMT joint complex intrinsically very stable. Between the medial cuneiform and second metatarsal base, there are 3 oblique ligaments (dorsal, interosseous, and plantar). These ligaments maintain the crucial osseous mortise for the second metatarsal base. The Lisfranc ligament is the interosseous ligament. This ligament is the strongest structure supporting the TMT joint complex, followed by the plantar ligament, and finally the dorsal ligament. ${ }^{11}$ Because of the relative strengths of the 3 ligaments, ligamentous disruption is thought to begin with the weaker dorsal ligament, followed by the plantar ligaments, and finally the Lisfranc ligament. $^{12}$ 


\section{Figure 2.}

A. Coronal CT Slice Through Lisfranc Joint Demonstrating Avulsion Type Fracture From Base of Second Metatarsal. B. Axial CT Slice. C. Sagittal CT Slice.
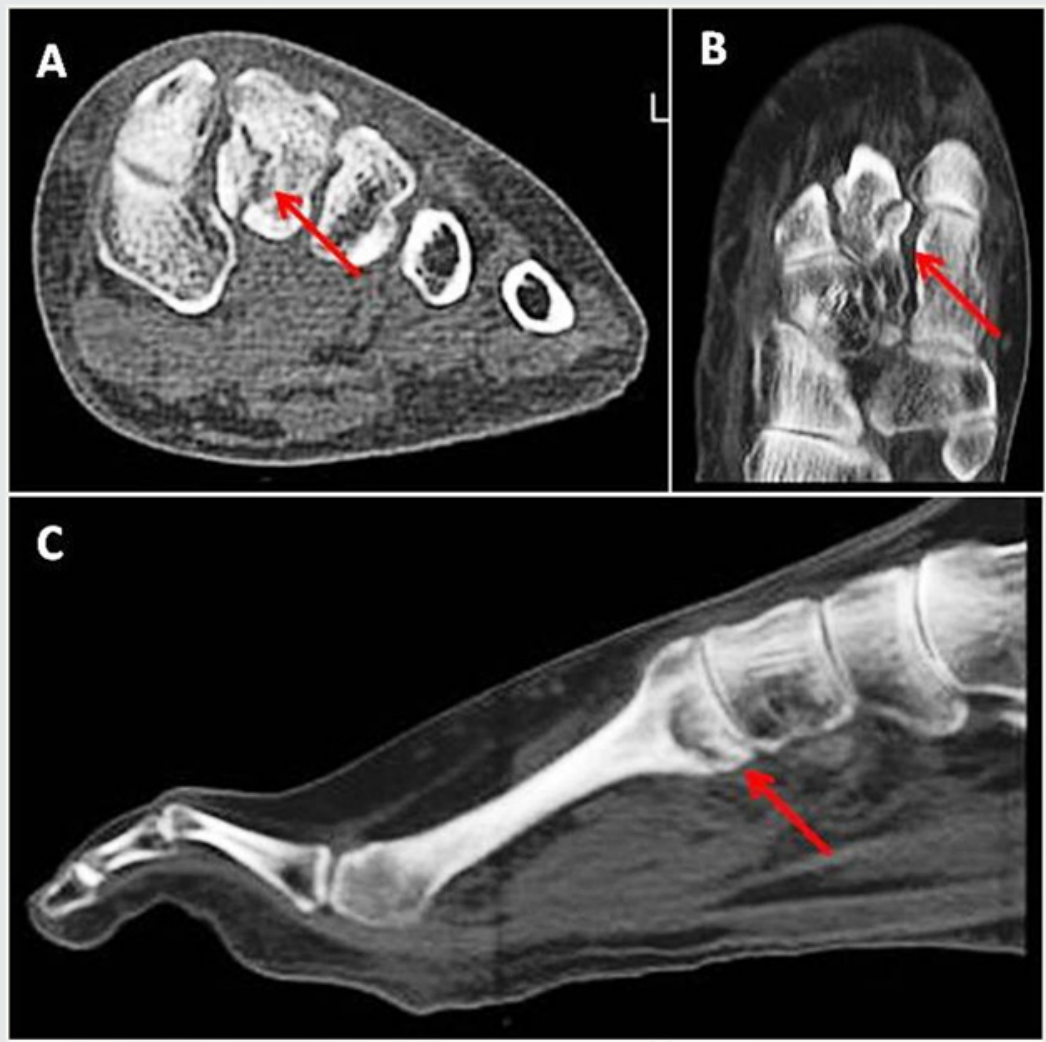

In the case presented, the fracture line seen on the coronal CT (Figure 2) appears to delineate the footprint of the insertion of the Lisfranc ligament at the medial base of the second metatarsal, as described by Solan et al. ${ }^{11}$ A weightbearing radiograph of the left foot demonstrates widening of the mortise confirming that the dorsal and plantar ligaments are also ruptured. This widening is not evident on non-weightbearing radiographs of the foot, but an avulsed fragment can clearly be seen. This fragment is more substantial than the "fleck" sign previously described by Myers. ${ }^{13}$ As described previously, ${ }^{12}$ this injury is consistent with a rupture of the dorsal oblique ligament, followed by rupture of the plantar oblique ligament, and then an avulsion fracture of the insertion of the Lisfranc ligament. We cannot find any documented case in the literature when this injury has occurred without injury or trauma. Most previous reports occur as repetitive strain injuries in ballet dancers and show the classical signs of stress fracture of normal initial radiographs followed by cortical reaction on subsequent investigation. We believe that this case of stress-avulsion fracture of the TMT joint complex is extremely pathology was investigated.

This patient's past medical history included APLS, SLE, and osteoporosis. Sangle et $\mathrm{a}^{14}$ reported on 19 cases of metatarsal stress fractures in female patients with SLE; 13 patients also had APLS. All these fractures occurred in the metatarsal shaft, and none involved the TMT joint complex. Also, 6 patients were found to have decreased bone mineral density, but the other 13 had normal bone density on DEXA scan. Only 5 uncommon, and possible contributing patients in this study had been taking oral steroid therapy for more than 6 months. The authors of this study found a link between metatarsal stress fractures and SLE and APLS but could not find a strong association with either decreased bone density or steroid use. They proposed a possible link between metatarsal stress fractures and microinfarcts within the bone. ${ }^{14}$ The authors postulated that microinfarcts are caused by a reaction between antiphospholipid antibodies and the endothelium, which leads to bone damage and fracture. ${ }^{15}$

Some authors have studied the association between warfarin and osteoporosis. ${ }^{16,17}$ A relationship between long-term warfarin therapy and osteoporotic fractures has been postulated. ${ }^{18}$ Warfarin works by interfering with the $\gamma$-carboxylation of glutamic acid residues on clotting factors II, VII, IX, and X. $\gamma$-Carboxylation of specific glutamic acid residues is also required for activation of osteocalcin and other bone matrix proteins. ${ }^{18}$ It has been hypothesized that warfarin may increase the risk of osteoporotic fractures. ${ }^{18}$ It remains unclear whether antithrombotic therapy plays a role in the development of metatarsal fractures. In the case series by Sangle et al, ${ }^{14} 2$ patients were on long-term warfarin at the time of their metatarsal fracture, but no definite association could be proven. That study has proposed a possible link between microinfarcts and metatarsal stress fractures, but the use of antithrombotic therapy would seem to be protective against the development of microinfarcts.

\section{Conclusion}

An increased risk of metatarsal stress fracture has been described in patients with APLS and SLE. An association between metatarsal stress fracture in patients with osteoporosis and in patients taking long-term warfarin has been proposed but not confirmed. We cannot, therefore, state any definite link between these conditions and metatarsal stress fractures, but we feel that in patients with any of these comorbidities 
who present with pain in the region of the Lisfranc joint complex with no history of trauma, it may be prudent to perform weight-bearing radiographs of the foot. Ongoing clinical suspicion in the absence of pathology on weightbearing radiographs warrants a CT scan to exclude stress fracture of the Lisfranc joint complex.FHS

\section{References}

1. Cassebaum WH. Lisfranc fracture dislocations. Clin Orthop Relat Res. 1963;30:116-129.

2. Hardcastle PH, Reschauer R, KutschaLissberg E, Schoffmann W. Injuries to the tarsometatarsal joint: incidence, classification and treatment. J Bone Joint Surg Br. 1982;64:349-356.

3. Mantas JP, Burks RT. Lisfranc injuries in the athlete. Clin Sports Med. 1994;13:719-730.

4. Trevino SG, Kodros S. Controversies in tarsometatarsal injuries. Orthop Clin North Am. 1995;26:229-238.

5. Vuori JP, Aro HT. Lisfranc joint injuries: Trauma mechanisms and associated injuries. J Trauma. 1993;35:40-45.
6. Curtis MJ, Myerson M, Szura B. Tarsometatarsal joint injuries in the athlete. Am J Sports Med. 1993;21: 497-502.

7. Albisetti W, Perugia D, De Bartolomeo O, Tagliabue L, Camerucci E, Calori GM. Stress fractures of the base of the metatarsal bones in young trainee ballet dancers. Int Orthop. 2010;17:108-113.

8. Sarimo J, Orava S, Alanen J. Operative treatment of stress fractures of the proximal second metatarsal. Scand J Med Sci Sports. 2007;17:383-386

9. Harrington T, Crichton KJ, Anderson IF.Overuse ballet injury of the base of the second metatarsal: a diagnostic problem. Am J Sports Med. 1993;21:591-598.

10. Micheli LJ, Sohn RS, Solomon R. Stress fracture of the second metatarsal involving Lisfranc's joint in ballet dancers: a new overuse injury of the foot. J Bone Joint Surg. 1985;67:1372-1375.

11. Solan MC, Moorman CT III, Miyamoto RG, Jasper LE, Belkoff SM. Ligamentous restraints of the second tarsometatarsal joint: a biomechanical evaluation. Foot Ankle Int. 2001;22:637-641.

12. Cain PR, Seligson D. Lisfranc's fracture dislocation with intercuneiform dislocation: presentation of two cases and a plan for treatment. Foot Ankle. 1981;2:156-160.

13. Meyer SA, Callaghan JJ, Albright JP, Crowley ET, Powell JW. Midfoot sprains in collegiate football players. Am J Sports Med. 1994:22:392-401.

14. Sangle S, D'Cruz DP, Khamashta MA, Hughes GR. Antiphospholipid antibodies, systemic lupus erythematosis, and nontraumatic metatarsal fractures. Ann Rheum Dis. 2004;63:1241-1243.

15. Rosenthal E, Sangle SR, Khamashta MA, D'Cruz D, Hughes DR. Orthopaedic manifestations of the antiphospholipid syndrome. Rev Med Interne. 2007;28:103-107.

16. Philip WJ, Martin JC, Richardson JM, Reid DM, Webster J, Douglas AS. Decreased axial and peripheral bone density in patients taking long term warfarin. QJM. 1995;88:635-640.

17. Jamal SA, Browner, Bauer DC, Cummings SR. Warfarin use and risk for osteoporosis in elderly women. Ann Intern Med. 1998;128:829-832.

18. Gage BF, Birman-Deych E, Radford MJ, Nilasena DS, Binder EF. Risk of osteoporotic fracture in elderly patients taking warfarin: results from the National Registry of Atrial Fibrillation 2. Arch Intern Med. 2006;166:241-246. 